Research Article

\title{
Shenlijia Attenuates Doxorubicin-Induced Chronic Heart Failure by Inhibiting Cardiac Fibrosis
}

\author{
Xutao Sun, ${ }^{1}$ Yunjia Song, ${ }^{1}$ Ying Xie, ${ }^{1}$ Jieru Han, ${ }^{1}$ Fei Chen, ${ }^{1}$ Yang Sun, \\ Bowen Sui $\mathbb{D}^{2}{ }^{2}$ and Deyou Jiang ${ }^{1}$ \\ ${ }^{1}$ School of Basic Medical Sciences, Heilongjiang University of Chinese Medicine, Harbin, China \\ ${ }^{2}$ Department of Pneumology, First Affiliated Hospital, Heilongjiang University of Chinese Medicine, Harbin, China
}

Correspondence should be addressed to Bowen Sui; suibowen2020@126.com

Received 29 December 2020; Revised 29 April 2021; Accepted 8 July 2021; Published 17 July 2021

Academic Editor: Yong-Bo Xue

Copyright (c) 2021 Xutao Sun et al. This is an open access article distributed under the Creative Commons Attribution License, which permits unrestricted use, distribution, and reproduction in any medium, provided the original work is properly cited.

Application of the anticancer drug doxorubicin (DOX) is restricted due to its adverse, cardiotoxic side effects, which ultimately result in heart failure. Moreover, there are a limited number of chemical agents for the clinical prevention of DOX-induced cardiotoxicity. Based on the theories of traditional Chinese medicine (TCM) on chronic heart failure (CHF), Shenlijia (SLJ), a new TCM compound, has been developed to fulfill multiple functions, including improving cardiac function and inhibiting cardiac fibrosis. In the present study, the protective effects and molecular mechanisms of SLJ on DOX-induced CHF rats were investigated. The CHF rat model was induced by intraperitoneal injection of DOX for six weeks with the cumulative dose of $15 \mathrm{mg} /$ $\mathrm{kg}$. All rats were then randomly divided into the control, $\mathrm{CHF}, \mathrm{CHF}+\mathrm{SLJ}(3.0 \mathrm{~g} / \mathrm{kg}$ per day), and CHF + captopril (3.8 mg/kg per day) groups and treated for further four weeks. Echocardiography and the assessment of hemodynamic parameters were performed to evaluate heart function. A protein chip was applied to identify proteins with diagnostic values that were differentially expressed following SLJ treatment. The data from these investigations showed that SLJ treatment significantly improved cardiac function by increasing the left ventricular ejection fraction, improving the hemodynamic index, and inhibiting interstitial fibrosis. Protein chip analysis revealed that SLJ upregulated MCP-1, MDC, neuropilin-2, TGF- $\beta 3$, thrombospondin, TIE-2, EG-VEGF/ PK1, and TIMP-1/2/3 expressions and downregulated that of MMP-13. In addition, immunohistochemistry and western blot results further confirmed that SLJ promoted TIMP-1/2/3 and inhibited MMP-13 expression. The results of the present study suggest that SLJ was effective against DOX-induced CHF rats and is related to the improvement of heart function and ultrastructure and the inhibition of myocardial fibrosis.

\section{Introduction}

Doxorubicin (DOX) is a broad-spectrum anthracycline anticancer agent that has significant effects on leukemia, lymphoma, and breast cancer [1]. Nevertheless, its clinical application is restricted on account of its dose-dependent and cumulative cardiotoxicity, which results in severe heart failure [2]. Once present, DOX-induced cardiotoxicity is irreversible and eventually develops into chronic heart failure (CHF). CHF is the end-stage manifestation of various heart diseases and is the most common cardiovascular disease. Its morbidity and prevalence are increasing worldwide, which is a relatively serious health problem [3].
Although mitochondrial oxidative stress, cardiomyocyte apoptosis, and the inflammatory response are involved in the development of DOX-induced cardiotoxicity, the precise molecular mechanisms require further clarification [4]. Traditionally, DOX-induced cardiotoxicity has been attributed to cardiomyocyte apoptosis due to the excessive production of activated oxygen in the mitochondria, which exceeds the regulatory function of the heart antioxidant defense system [5]. However, endothelial dysfunction, vascular damage, and myocardial fibrosis represent important damage mechanisms to cardiac function following DOX therapy [6-9]. In this regard, the identification of novel therapeutics to alleviate cardiac fibrosis is urgently required. 
Over the last few centuries, traditional Chinese medicine (TCM) has been used to regulate homeostasis in the human body, and Chinese herbal medicines have become ubiquitous for the treatment of specific diseases. Some have cardiovascular effects and can be used in combination with pharmaceutical drugs. Moreover, in the last few decades, TCM compounds have been widely accepted as treatments of cardiovascular diseases in China, as they exert multiple targeted and comprehensive conditioning effects $[10,11]$. In TCM, it is believed that the heart Qi is the power that maintains blood circulation and keeps blood in the vascellum. Heart failure is primarily caused by Qi deficiency, followed by blood stasis and drowning. To relieve heart failure, it is necessary to restore the heart Qi, reactivate the blood, and disinhibit water flow [12]. The heart is physiologically associated with and thus can pathologically influence other organs during heart failure; therefore, the treatment principles of TCM are to accelerate blood circulation, disinhibit water flow, and disperse swelling by invigorating the heart $\mathrm{Qi}$ and warming the Yang.

The SLJ formula was developed from a well-known capsule of Tingli Dazao, according to the principles of TCM. SLJ contains the following seven commonly used herbs: Ren Shen, Hong Jing Tian, Ting Li Zi, Wu Jia Pi, San Qi, Shan Zhu $\mathrm{Yu}$, and Xian Ling Pi. Among these herbs, based on the Chinese Pharmacopoeia [13], Ginseng is a classical TCM for supplying Qi and warming the Yang, and Rhodiola enhances these effects. Sanchi is often used for promoting blood circulation, removing blood stasis, and relieving pain and swelling. Semen Lepidii has the effect of dispelling phlegm and improving edema. Acanthopanax is traditionally used for dispelling wind, improving kidney function, strengthening the bones, and removing blood stasis. Epimedium nourishes the kidney Yang, reinforces the muscles and bones, and relieves rheumatism. Finally, Macrocarpium fills the blood and replenishes the liver and kidneys. In addition, the bioactivity and pharmacological actions of these herbs have been scientifically studied in the past decades. Ginseng has been reported to improve cardiac function, relieve arrhythmia, and alleviate apoptosis associated with heart failure [14]. Additionally, Ginseng relieves DOX-induced cardiotoxicity by inhibiting apoptosis in mice, demonstrating its potential for the treatment of DOX-induced myocardial toxicity in a clinical setting [15]. Rhodiola is commonly prescribed for protecting cardiomyocytes against hypoxiainduced necrosis and apoptosis [16], and Sanchi is known to promote angiogenesis and prevent oxidative stress and apoptosis in the ischemic myocardium, both in vitro and in vivo $[17,18]$. Semen Lepidii is one of the most commonly used herbs for $\mathrm{CHF}$, which increases pulse pressure and cAMP efflux in a dose-dependent manner [19]. Acanthopanax has shown remarkable anti-hypertensive and anti-inflammatory effects [20], while Macrocarpium possesses antiarrhythmia and antioxidant properties [21]. Additionally, Epimedium is widely used in the clinic to stimulate angiogenesis, prevent endoplasmic reticulum stress, and inhibit left ventricular dysfunction, myocardial remodeling, and apoptosis in CHF rats [22-24].
The multiple pharmacological effects of each herb in the SLJ capsule are thought to be effective for the treatment of $\mathrm{CHF}$ and include improving cardiac function, reducing myocardial apoptosis, inhibiting ventricular remodeling, and promoting neovascularization. Therefore, it was speculated that the SLJ formula was likely to be cardioprotective in DOX anti-tumor treatment, which may provide a new means of clinically alleviating DOX-induced cardiotoxicity and CHF. However, there is currently insufficient evidence to support these claims. In the present study, an experimental DOX-induced CHF rat model was constructed to investigate the potential effects and possible underlying mechanisms of SLJ on CHF.

\section{Materials and Methods}

2.1. SLJ Preparation. As shown in Table 1, the seven medicinal components of SLJ were purchased from the Pharmacy of the First Affiliated Hospital of Heilongjiang University of Chinese Medicine. The herbs were combined in a TCM decoction container, soaked in $12.5 \mathrm{v} / \mathrm{w}$ distilled water for $30 \mathrm{~min}$, boiled for $60 \mathrm{~min}$, and extracted 3 times. The extract was filtrated using a G4 filter and concentrated in a $60^{\circ} \mathrm{C}$ water bath. The final extract $(3 \mathrm{~g} / \mathrm{mL})$ was pasteurized, bottled, and stored at $4^{\circ} \mathrm{C}$.

2.2. HPLC Fingerprint. One gram of SLJ capsule was sonicated in $10 \mathrm{~mL}$ of ethyl alcohol. The extract was passed through a $0.45 \mu \mathrm{m}$ PTFE filter before the HPLC injection. After that, the supernatant was collected and analyzed for chemical fingerprint analysis with Agilent HPLC 1200 system (Agilent, Germany). The samples were achieved on a PLATISIL C18 column $(4.6 \mathrm{~mm} \times 250 \mathrm{~mm} ; 5 \mu \mathrm{m})$. The detection wavelength was set to $280 \mathrm{~nm}$, and the flow rate was $0.8 \mathrm{~mL} / \mathrm{min}$ [25].

2.3. Animals. Male Wistar rats weighing $180-200 \mathrm{~g}$ were purchased from Liaoning Changsheng Biotechnology Co., Ltd. (Animal license number: SCXK (liao) 2015-0001). The animals were raised with standard food and water at $25 \pm 1{ }^{\circ} \mathrm{C}$ and $50 \pm 5 \%$ humidity, with a $12 \mathrm{~h}$ light/dark cycle. All animal testing procedures were conducted in accordance with the Ethics Committee Guidelines on the Care and Use of Laboratory Animals (NIH Publications, no. 85-23, revised 1996). The $\mathrm{CHF}$ rats were established by intraperitoneal injection of DOX $(2.5 \mathrm{mg} / \mathrm{kg}$ body weight $)$ once a week for six weeks [26]. Following the last injection, 6 rats from the control and DOX groups were randomly selected to evaluate cardiac function by echocardiography. Compared with the control group, DOX group ejection fraction (EF) and shortening fraction (FS) were significantly reduced, and left ventricular end-diastolic and end-systolic internal diameters (LVIDd/LVIDs) were significantly higher in the DOX group, indicating that the DOX-induced $\mathrm{CHF}$ rat model was successfully constructed [27]. Subsequently, all rats were randomly assigned to the control, model, SLJ (3.0 $\mathrm{g} / \mathrm{kg}$ per day), or captopril treatment group $(3.8 \mathrm{mg} / \mathrm{kg}$ per day). The model and control group received equal 
TABLe 1: Components of SLJ.

\begin{tabular}{|c|c|c|}
\hline Number & Identification & Formula \\
\hline \multirow[t]{2}{*}{1} & Heterodendrin & $\mathrm{C}_{11} \mathrm{H}_{19} \mathrm{O}_{6}$ \\
\hline & Isoquercitrin & $\mathrm{C}_{21} \mathrm{H}_{20} \mathrm{O}_{12}$ \\
\hline \multirow[t]{2}{*}{2} & Ginsenoside Rb1 & $\mathrm{C}_{54} \mathrm{H}_{92} \mathrm{O}_{23}$ \\
\hline & Rosmarinic acid & $\mathrm{C}_{18} \mathrm{H}_{16} \mathrm{O}_{8}$ \\
\hline \multirow[t]{2}{*}{3} & p-Cymene & $\mathrm{C}_{10} \mathrm{H}_{16}$ \\
\hline & Ginsenoside $\mathrm{Rg} 1+\mathrm{O}$ & $\mathrm{C}_{42} \mathrm{H}_{72} \mathrm{O}_{15}$ \\
\hline \multirow[t]{2}{*}{4} & 20(R)-Ginsenoside F1 & $\mathrm{C}_{36} \mathrm{H}_{62} \mathrm{O}_{9}$ \\
\hline & Descurainolide B & $\mathrm{C}_{21} \mathrm{H}_{22} \mathrm{O}_{8}$ \\
\hline 5 & $\gamma$-Terpinene & $\mathrm{C}_{10} \mathrm{H}_{16}$ \\
\hline \multirow[t]{2}{*}{6} & $7 \alpha$-O-Methyl morroniside & $\mathrm{C}_{18} \mathrm{H}_{28} \mathrm{O}_{11}$ \\
\hline & 20(R)-Ginsenoside Rh1 & $\mathrm{C}_{36} \mathrm{H}_{62} \mathrm{O}_{9}$ \\
\hline \multirow[t]{2}{*}{7} & Gallic acid & $\mathrm{C}_{7} \mathrm{H}_{6} \mathrm{O}_{5}$ \\
\hline & Ginsenoside $\operatorname{Rg} 1+2$ glc & $\mathrm{C}_{54} \mathrm{H}_{92} \mathrm{O}_{24}$ \\
\hline 8 & Ginsenoside Rg6 & $\mathrm{C}_{42} \mathrm{H}_{70} \mathrm{O}_{12}$ \\
\hline \multirow[t]{3}{*}{9} & Magnoflorine & $\mathrm{C}_{20} \mathrm{H}_{24} \mathrm{O}_{4}$ \\
\hline & Ginsenoside Rg1-glc & $\mathrm{C}_{36} \mathrm{H}_{62} \mathrm{O}_{9}$ \\
\hline & d-Limonene & $\mathrm{C}_{10} \mathrm{H}_{16}$ \\
\hline \multirow[t]{2}{*}{10} & Ginsenoside Rg1-glc isomer & $\mathrm{C}_{36} \mathrm{H}_{62} \mathrm{O}$ \\
\hline & $7 \alpha$-O-Ethylmorroniside & $\mathrm{C}_{19} \mathrm{H}_{30} \mathrm{O}_{11}$ \\
\hline 11 & Ginsenoside $\mathrm{Rg} 1-\mathrm{H}_{2} \mathrm{O}$ isomer & $\mathrm{C}_{36} \mathrm{H}_{60} \mathrm{O}_{8}$ \\
\hline \multirow[t]{2}{*}{12} & Protopanaxatriol & $\mathrm{C}_{30} \mathrm{H}_{52} \mathrm{O}_{4}$ \\
\hline & 4-Terpineol & $\mathrm{C}_{10} \mathrm{H}_{18} \mathrm{O}$ \\
\hline 13 & Ginsenoside Rb1-3glc & $\mathrm{C}_{36} \mathrm{H}_{62} \mathrm{O}_{8}$ \\
\hline \multirow[t]{2}{*}{14} & p-Hydroxyphenacyl- $\beta$-D-glucopyranoside & $\mathrm{C}_{14} \mathrm{H}_{18} \mathrm{O}_{8}$ \\
\hline & Ginsenoside $\mathrm{Rb} 1-4 \mathrm{glc}+\mathrm{O}$ & $\mathrm{C}_{30} \mathrm{H}_{52} \mathrm{O}_{4}$ \\
\hline 15 & trans-Nerolidol & $\mathrm{C}_{15} \mathrm{H}_{26} \mathrm{O}$ \\
\hline 16 & Thunbergene & $\mathrm{C}_{20} \mathrm{H}_{32}$ \\
\hline \multirow[t]{2}{*}{17} & n-Hexadecanoic acid & $\mathrm{C}_{16} \mathrm{H}_{32} \mathrm{O}_{2}$ \\
\hline & Sagittatoside A & $\mathrm{C}_{33} \mathrm{H}_{40} \mathrm{O}_{15}$ \\
\hline 18 & Sagittatoside B & $\mathrm{C}_{32} \mathrm{H}_{38} \mathrm{O}_{14}$ \\
\hline \multirow[t]{2}{*}{19} & 2-Phenylethyl-1- $\beta$-D-glucoside & $\mathrm{C}_{14} \mathrm{H}_{20} \mathrm{O}_{6}$ \\
\hline & Epimedoside & $\mathrm{C}_{37} \mathrm{H}_{44} \mathrm{O}_{17}$ \\
\hline \multirow[t]{2}{*}{20} & Epimedigrandioside A & $\mathrm{C}_{39} \mathrm{H}_{46} \mathrm{O}_{18}$ \\
\hline & 12-Octadecadienoic acid & $\mathrm{C}_{18} \mathrm{H}_{32} \mathrm{O}_{2}$ \\
\hline 21 & Ethyl gallate & $\mathrm{C}_{9} \mathrm{H}_{10} \mathrm{O}_{5}$ \\
\hline 22 & Anhydroicaritin & $\mathrm{C}_{21} \mathrm{H}_{20} \mathrm{O}_{6}$ \\
\hline 23 & Desmethylanhydroicaritin & $\mathrm{C}_{20} \mathrm{H}_{18} \mathrm{O}_{6}$ \\
\hline
\end{tabular}

volumes of $0.9 \%$ physiological saline. All drugs (including saline) were administered intragastrically once daily for four weeks.

2.4. Echocardiography. Echocardiography and subsequent analysis were performed by the same experienced researcher in a blinded manner. Following anesthetization with intraperitoneal injection of $3 \%$ sodium pentobarbital solution $(30 \mathrm{mg} / \mathrm{kg})$, the rats were fixed in a low-motion position, and the long axis of the parasternal left ventricular was located with a 7-12 MHz phased-array transducer (Vivid 7; GE Healthcare). Detection indexes included the LVIDd/LVIDs, $\mathrm{EF}$, and FS. The average of $\geq 3$ measurements was calculated for each rat.

2.5. Hemodynamics. While the animals were still anesthetized, a tip-transducer catheter (diameter, $1 \mathrm{~mm}$ ) was inserted into the right common carotid artery to assess the following hemodynamic parameters: left ventricular end- diastolic pressure (LVEDP), left ventricular systolic pressure (LVSP), maximum pressure positive, and negative velocity ( $\pm \mathrm{dp} / \mathrm{dtmax})$. The results were then analyzed using PowerLab software (30 series; ADInstruments Shanghai Trading Co.). After hemodynamic measurements were acquired, the rats were then euthanized by anesthetic overdose. The hearts were rapidly removed, divided into two parts at the midpoint of the left ventricular long axis, and reserved for histological analysis.

2.6. Myocardium Tissue Processing. After the above experiment, part of the apical myocardium was excised and fixed with formalin for histomorphological assessment. Rat's left ventricular myocardium tissues were lysed with lysis buffer (50 mM Tris base, $1 \mathrm{mM}$ EDTA, $150 \mathrm{mM} \mathrm{NaCl}, 1 \% \mathrm{NP}-400$, $25 \%$ sodium deoxycholate, protease inhibitor cocktail, and $\mathrm{PH}$ 7.4). Protein concentration was determined using a BCA protein assay kit (Pierce; Thermo Fisher Scientific, Inc.) according to the manufacturer's instructions.

2.7. Transmission Electron Microscopy. To analyze the effect of SLJ on the heart tissue ultrastructure, transmission electron microscopy was performed. Fresh myocardial tissue samples from the apex cordis were fixed overnight with $2.5 \%$ glutaraldehyde at $4^{\circ} \mathrm{C}$ and washed 3 times in PBS. The tissues were dehydrated and embedded in epoxy resin and subsequently solidified at $60^{\circ} \mathrm{C}$ for $2 \mathrm{~h}$. Each heart was cut into 2 ultrathin sections and stained with $0.2 \%$ lead citrate and $1 \%$ uranyl acetate. Images were obtained at different magnifications using a JEM-1400PLUS transmission electron microscope (JEOL, Ltd.).

2.8. Hematoxylin-Eosin (HE) Staining. Myocardial specimens were rinsed with phosphate buffer saline (PBS) and fixed overnight with $4 \%$ paraformaldehyde at $4^{\circ} \mathrm{C}$. The specimens were then dehydrated with $75 \%, 95 \%$, and $100 \%$ alcohol, treated with xylene, and embedded in paraffin. $\mathrm{HE}$ staining was performed using serial tissue sections $(4 \mu \mathrm{m}$ each), and histopathological changes to the myocardial tissues were evaluated with an optical microscope $(\times 400)$.

2.9. Antibody Arrays. The relative expression levels of 90 proteins extracted from the myocardial tissues of the control, model, and SLJ group were detected using cytokine antibody arrays (Rat Antibody L-Series 90 Array) in accordance with the manufacturer's protocol. Briefly, chips containing 90 different antibodies were sealed with blocking buffer and subsequently incubated with the protein samples. The chips were then washed and incubated with biotin-labeled antibodies against these different proteins. Subsequently, the chips were incubated with a horseradish peroxidase-labeled anti-streptomycin biotin antibody, developed with enhanced chemiluminescence reagents (Cell Signaling Technology, Inc.), and exposed to autoradiographic film (BioMax Lite; Kodak). The films were scanned, and the images were converted to pixel densities; relative protein expression was 
analyzed by subtracting the background intensities and comparing these with the positive control spots.

2.10. Immunohistochemistry. Immunohistochemical staining was performed to detect the protein expression levels of collagen I, collagen III, matrix metalloproteinase MMP-13, and metalloproteinase inhibitors TIMP-1, TIMP-2, and TIMP-3. Tissues were embedded as described above. The paraffin-embedded myocardial tissues were cut into $4 \mu \mathrm{m}$ sections and placed on slides. The sections were deparaffinized, dehydrated, and incubated in $3 \% \mathrm{H}_{2} \mathrm{O}_{2}$ at $25^{\circ} \mathrm{C}$ for $10 \mathrm{~min}$. Following incubation with pepsin at $37^{\circ} \mathrm{C}$ for $30 \mathrm{~min}$ to restore antigen activity, blocking was performed with $5 \%$ goat serum (Invitrogen, Co., USA) at room temperature for $30 \mathrm{~min}$. Next, the sections were incubated with primary antibodies rabbit anti-collagen I, MMP-13, TIMP-3 (1:500; Abcam, USA), mouse anti-collagen III (1:1,000; Abcam, USA), TIMP-1 (1:500; Abcam, USA), and TIMP-2 (1:800; Abcam, USA), overnight at $4^{\circ} \mathrm{C}$. On the second day, the sections were washed in PBS for $5 \mathrm{~min}$ and subsequently incubated with corresponding secondary antibodies (concentration dilution is twice that of the primary antibody) at $37^{\circ} \mathrm{C}$ for $1 \mathrm{~h}$. The tissue slices were washed with PBS $(3 \times 5 \mathrm{~min})$ and counterstained with hematoxylin before washing thoroughly. Images were obtained using an optical microscope at $\times 400$ magnification.

2.11. Western Blot. Western blot was performed to determine the levels of collagen I, collagen III, TIMP-1/2/3, and MMP-13 in rat myocardium. The myocardium was ground into a homogenate with RIPA lysis buffer (the mass volume ratio $1: 10(\mathrm{mg} / \mu \mathrm{L}))$, and then the homogenate was centrifuged for $15 \mathrm{~min}$ at $4^{\circ} \mathrm{C}$ to separate the supernatant [28], and the protein concentration was detected by BCA Kit (Beijing Biyuntian Co., China). The same amount of protein was separated by boiling with $10 \%$ SDS-PAGE. The protein gels were then removed to the nitrocellulose membrane by electrophoresis (Amersham, USA). The protein bands were incubated with the primary antibodies for mouse anti-collagen III (1:500; Sigma, USA), MMP-13 (1:400; Sigma, USA), GAPDH (1:2,000; Kangcheng, China), rabbit anti-collagen I (1:1,000; Abcam, USA), TIMP-1 (1:500; Abcam, USA), TIMP-2 (1:1,000; Abcam, USA), and TIMP-3 (1:1,000; CST, USA). The bands were then washed and incubated with the corresponding secondary antibodies (1:3,000; CST, USA) for $1 \mathrm{~h}$. Bands were visualized on a FluorChem $M$ MultiFluor System (ProteinSimple, USA) using chemiluminescence detection reagents. Optical density analysis was done with AlphaEaseFC (Alpha, USA).

2.12. Statistical Analysis. All statistical analyses were performed using SPSS 17.0 (SPSS, Inc.). One-way ANOVA was used following Tukey's test to compare the differences among four groups, and the Mann-Whitney $U$ test was used to compare group differences in the protein tissue array results. $P<0.05$ was considered to indicate a statistically significant difference.

\section{Results}

3.1. Fingerprint of SLJ. HPLC chromatograms of $95 \%$ ethanol extract of SLJ were obtained, in which there were mainly 23 eluted peaks being identified (Figure 1). The 23 peaks represented major constituents of SLJ extracts with consistent retention values (RSDs of retention times lower than $1 \%$ and those of most peak areas lower than $8 \%$ ). The authenticated 23 peaks, including 37 components of SLJ, were confirmed by comparing retention times with the chemical standards (Table 2).

\subsection{SLJ Alleviates DOX-Induced Cardiac Dysfunction.} Cardiac dysfunction was induced by intraperitoneal administration of DOX $(2.5 \mathrm{mg} / \mathrm{kg} / \mathrm{w})$ for six weeks, followed by SLJ, captopril, or saline, administered intragastrically for a further four weeks. Echocardiography and the assessment of hemodynamic parameters revealed significant impairment in left ventricular function after DOX infusion, as indicated by increased LVIDd, LVIDs, and LVEDP and decreased EF, FS, LVSP, +dp/dtmax, and -dp/dtmax compared with the control group (Figure 2). Furthermore, SLJ and captopril significantly ameliorated myocardial dysfunction and remodeling, which was verified by increased EF, FS, LVSP, +dp/dtmax, and -dp/dtmax as well as decreased LVIDd, LVIDs, and LVEDP compared with the model group (Figure 2). These results indicate that SLJ treatment improves cardiac function in DOX-induced CHF.

3.3. SLJ Preserves the Myocardial Ultrastructure in Rats with DOX-Induced CHF. Transmission electron microscopy was performed to assess the myocardial ultrastructure of DOXinduced CHF rats (Figure 3(a)). Compared with the control group, the sarcomeres were destroyed, and the mitochondria became swollen and damaged in the CHF group; treatment with SLJ and captopril resulted in an improvement to the myocardial ultrastructure.

3.4. SLJ Inhibits Inflammation and Fibrosis in Rats with DOX-Induced CHF. As shown in Figure 3(b), the model group elicited significantly increased inflammatory-cell infiltration in comparison with the control group, which was reduced by SLJ treatment. Collagens I and III, the primary collagen isoforms, were produced by cardiac fibroblasts. Representative immunohistochemical images and western blot showed that collagen I and III expressions in the left ventricle sections were decreased following SLJ and captopril treatment, compared with those of the model group (Figures 3(c)-3(f)). These findings indicate that SLJ suppresses inflammation and fibrosis in rats with DOX-induced CHF.

3.5. Antibody Microarray Analysis. The expression levels of 90 known proteins (including cytokines, inflammatory factors, chemokines, MMPs, growth factors, and soluble receptors) were measured in the myocardial tissues of all rats. The results showed that 8 proteins were significantly 


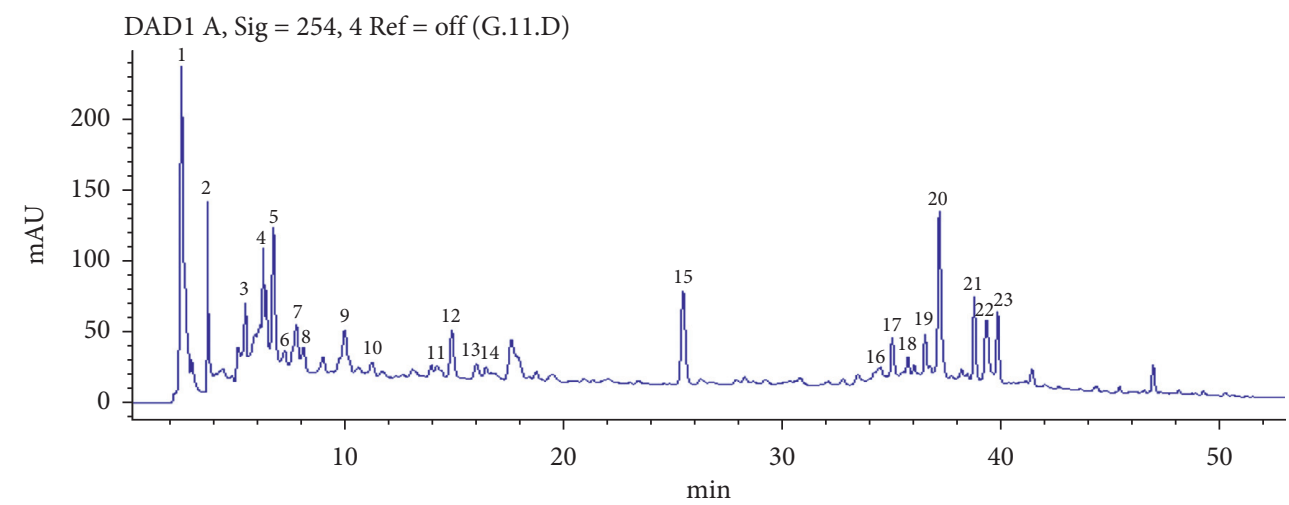

FIgURE 1: Fingerprint of SLJ. There were mainly 23 eluted peaks being identified.

Table 2: Composition of Shenlijia decoction.

\begin{tabular}{lccc}
\hline Chinese name & Latin name & English name & Quantity $(\mathrm{g})$ \\
\hline Ren Shen & Panax ginseng & Ginseng & 30 \\
Hong Jing Tian & Rhodiola rosea & Rhodiolae crenulatae & 15 \\
Ting Li Zi & Semen lepidii & Seed of pepperweed & 20 \\
Wu Jia Pi & Acanthopanax senticosus & Slenderstyle acanthopanax bark & \\
San Qi & Panax notoginseng & Notoginseng radix et rhizoma & \\
Shan Zhu Yu & Fructus corni & Common macrocarpium fruit & 15 \\
Xian Ling Pi & Epimedii folium & Epimedium & 15 \\
\hline
\end{tabular}

Control
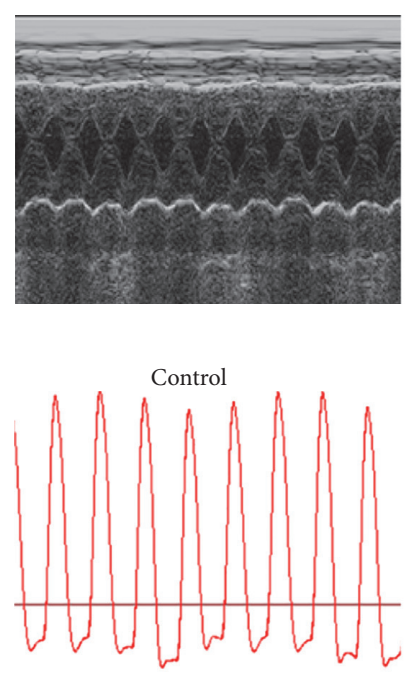

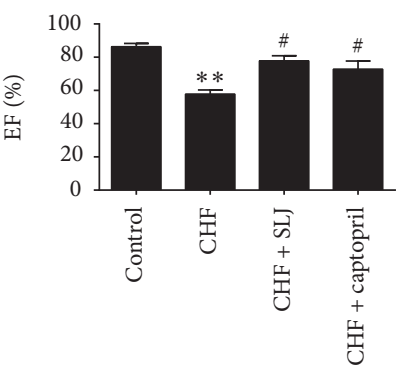

CHF

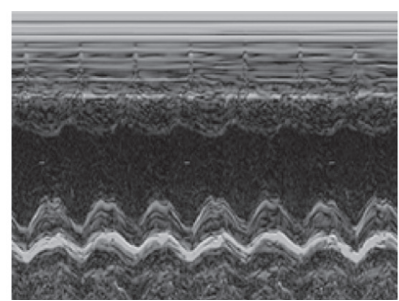

CHF

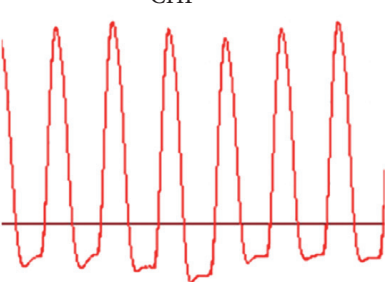

$\mathrm{CHF}+\mathrm{SLJ}$

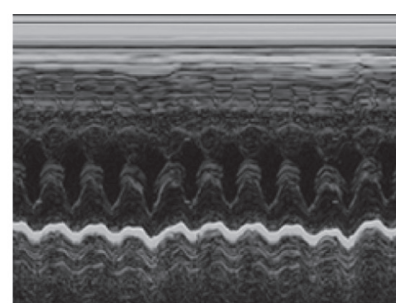

(a)

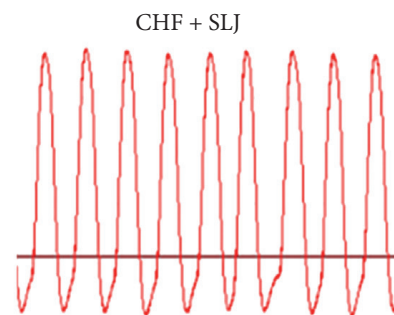

CHF + captopril

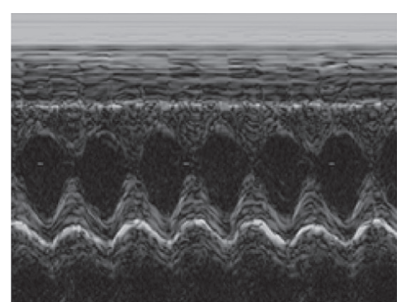

$\mathrm{CHF}+$ captopri

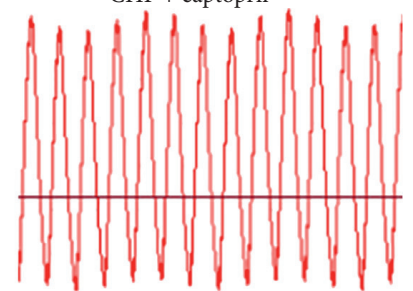

(b)
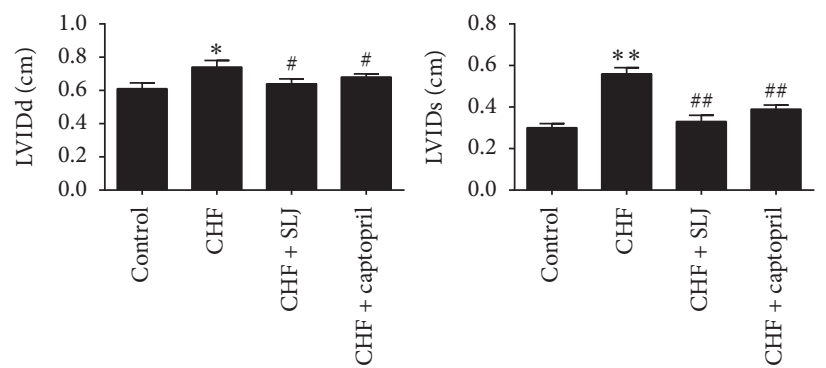

(c)

FIgURE 2: Continued. 


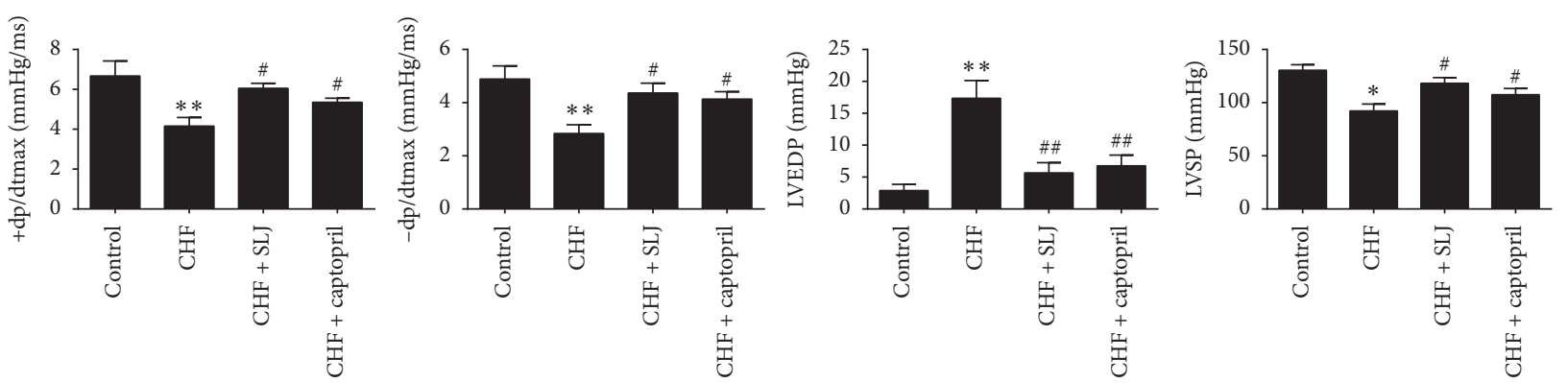

(d)

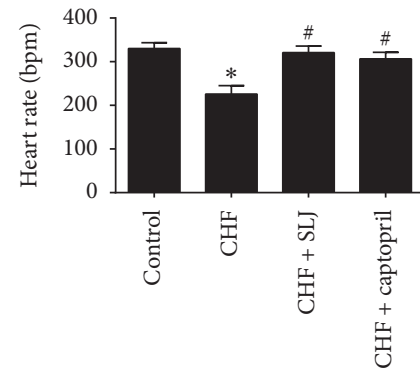

(e)

FIgURE 2: Comparison of echocardiographic and hemodynamic parameters between the control and experimental groups following treatment with SLJ: (a, b) M-mode echocardiographic and hemodynamic images for each group; (c) cardiac systolic functions indicated by EF, FS, LVIDd, and LVIDs were compared among different groups; and (d) comparison of LVSP, LVEDP, $+\mathrm{dp} / \mathrm{dtmax}$, and $-\mathrm{dp} / \mathrm{dtmax}$ hemodynamic values among different groups after treatment with SLJ. Data are expressed as the mean \pm SE. $n=8 .{ }^{*} P<0.05$ and ${ }^{* *} P<0.01$ vs. the control group. ${ }^{\#} P<0.05$ and ${ }^{\# \#} P<0.01$ vs. the CHF group. SJL, Shenlijia; EF, ejection fraction; FS, shortening fraction; LVIDd, left ventricular end-diastolic internal diameter; LVIDs, left ventricular end-systolic internal diameter; LVSP, left ventricular systolic pressure; LVEDP, left ventricular end-diastolic pressure; $+\mathrm{dp} / \mathrm{dtmax}$, maximum pressure positive velocity; $-\mathrm{dp} / \mathrm{dtmax}$, maximum pressure negative velocity; and $\mathrm{CHF}$, chronic heart failure.

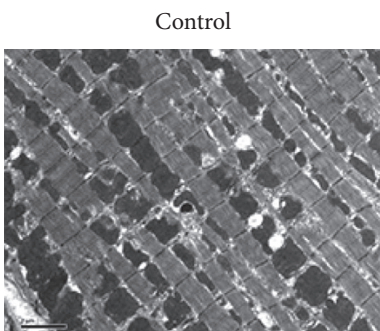

Control

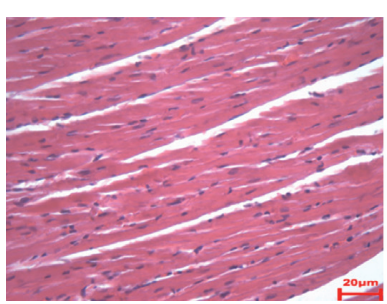

$\mathrm{CHF}$

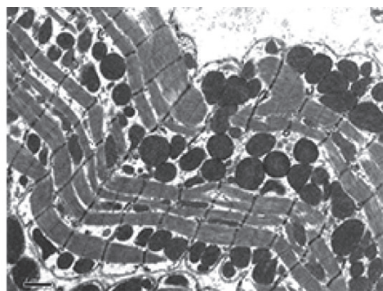

(a)

CHF

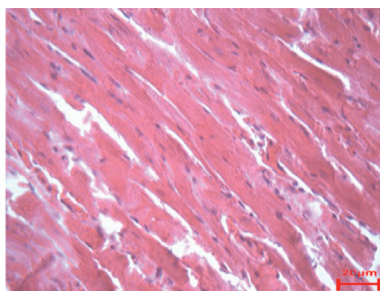

$\mathrm{CHF}+\mathrm{SLJ}$

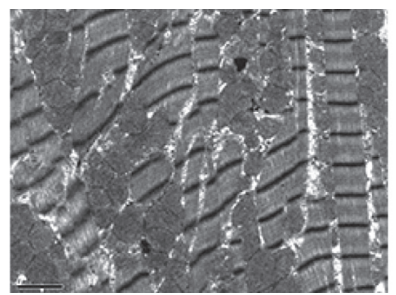

$\mathrm{CHF}+\mathrm{SLJ}$

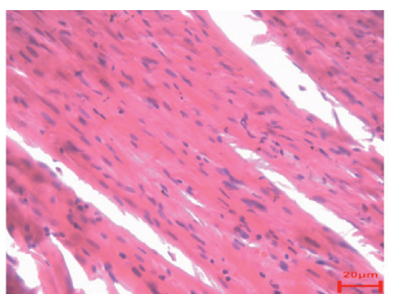

$\mathrm{CHF}+$ captopril

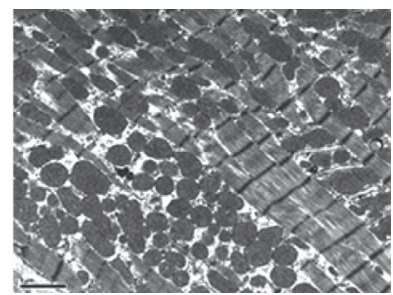

CHF + captopril

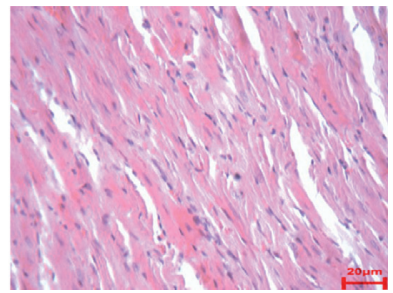

(b)

Figure 3: Continued. 
Control

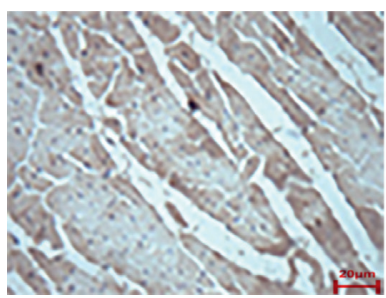

Control

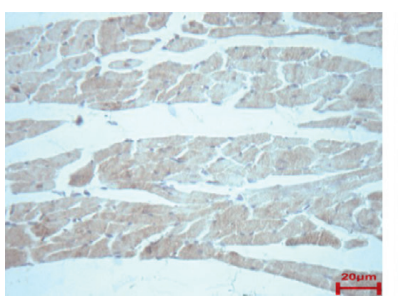

CHF

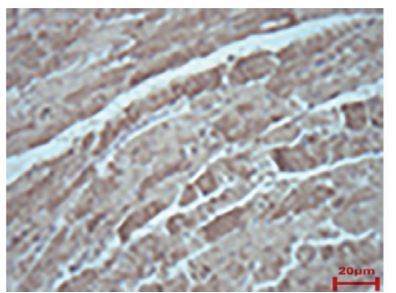

$\mathrm{CHF}+\mathrm{SLJ}$

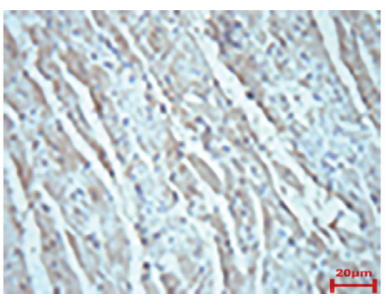

(c)
$\mathrm{CHF}$

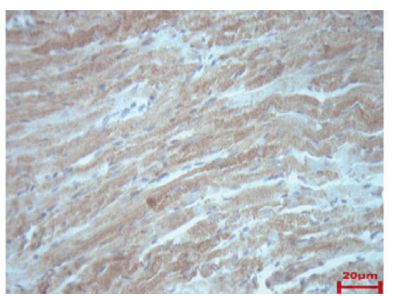

$\mathrm{CHF}+\mathrm{SLJ}$

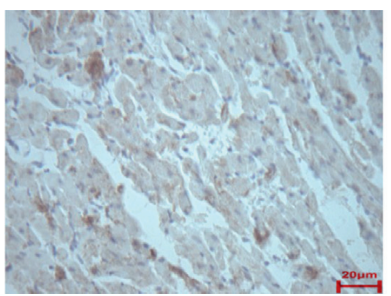

CHF + captopril

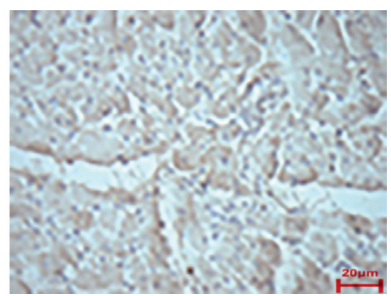

$\mathrm{CHF}+$ captopril

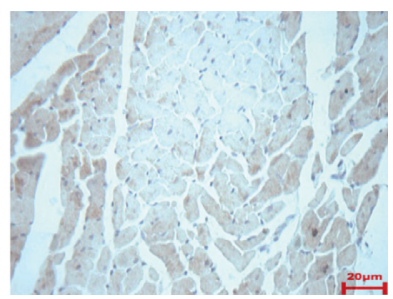

(d)
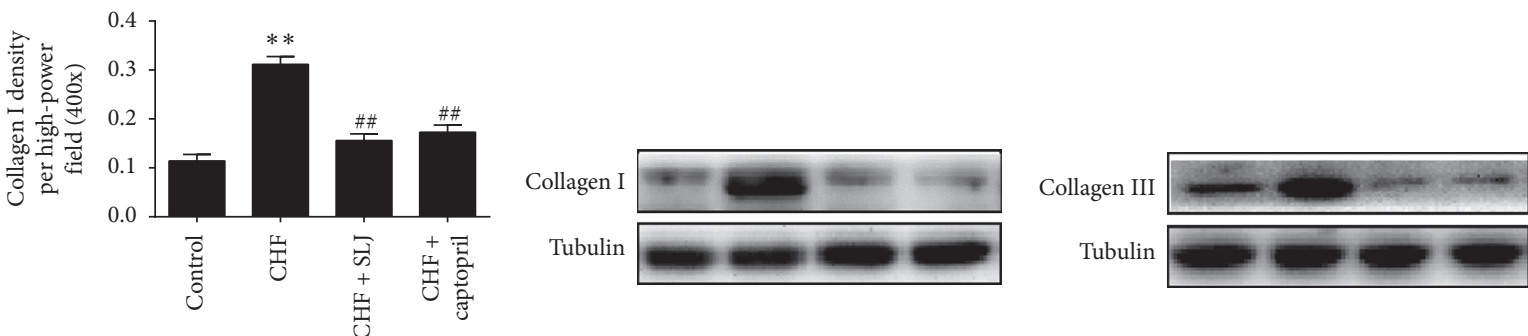

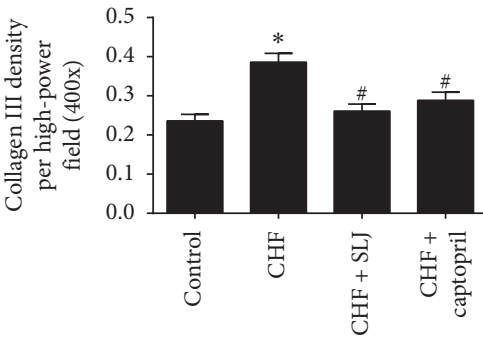

(e)

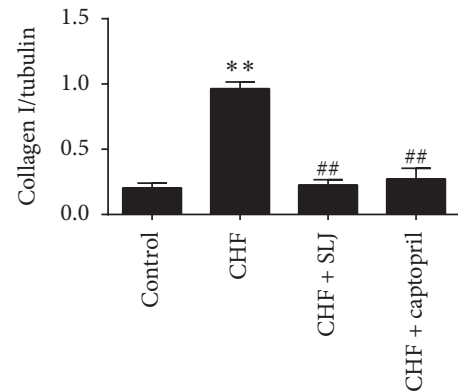

(f)

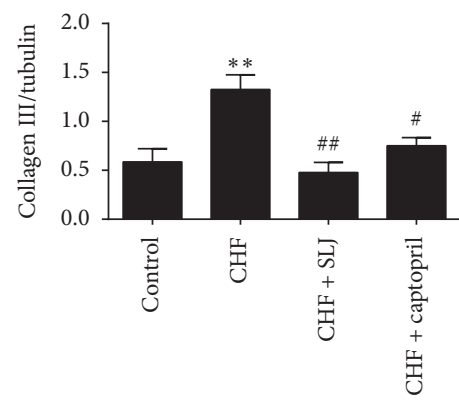

(g)

FIGURE 3: SLJ protects the myocardial ultrastructure and reduces inflammation and fibrosis: (a) representative transmission electron microscopy ( $\times 5000)$ images of the left ventricle; (b) HE staining for inflammatory-cell counts; (c, d) expression of collagen I and III protein in the myocardium using immunohistochemistry; $(\mathrm{e}, \mathrm{f})$ western blot analysis of collagen I and III protein expression in myocardium. $n=8$. ${ }^{*} P<0.05$ and ${ }^{* *} P<0.01$ vs. the control group. ${ }^{\#} P<0.05$ and ${ }^{\# \#} P<0.01$ vs. the CHF group. SJL, Shenlijia; HE, hematoxylin-eosin; and CHF, chronic heart failure.

upregulated and 28 were significantly downregulated in the CHF group, compared with the control group. In addition, 14 proteins were significantly upregulated, and 11 were significantly downregulated in the SLJ treatment group, compared with the CHF group (Figure 4(a)). Furthermore, the results of these intergroup comparisons were again compared with each other, revealing that 11 proteins exhibited significantly different expression levels following treatment with CHF with SLJ. Similarities in the abundance of these 11 proteins were sorted by a clustering algorithm, and 3 principal clusters were identified for the control group, and the CHF and SLJ treatment groups, respectively (Figures 4(b) and 4(c)).
3.6. SLJ Regulates the Balance between TIMP-1, TIMP-2, $T I M P-3$, and $M M P-13$. It has been clearly demonstrated that the imbalance between MMPs and their endogenous inhibitors TIMPs plays an important role in left ventricular remodeling. In the present study, analysis of the protein chip results indicated that the expression of MMP-13 was increased in the CHF group, compared with the control group, which was reversed by SLJ and captopril treatment; the expressions of TIMP-1, TIMP-2, and TIMP-3 were the opposite to that of MMP-13. Furthermore, the results of subsequent immunohistochemistry and western blot were consistent with these data (Figure 5). These analyses indicate that SLJ inhibits cardiac remodeling, potentially by 

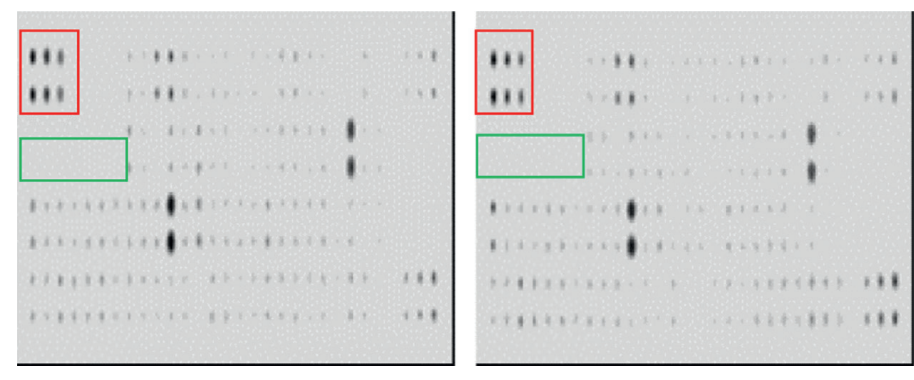

(a)
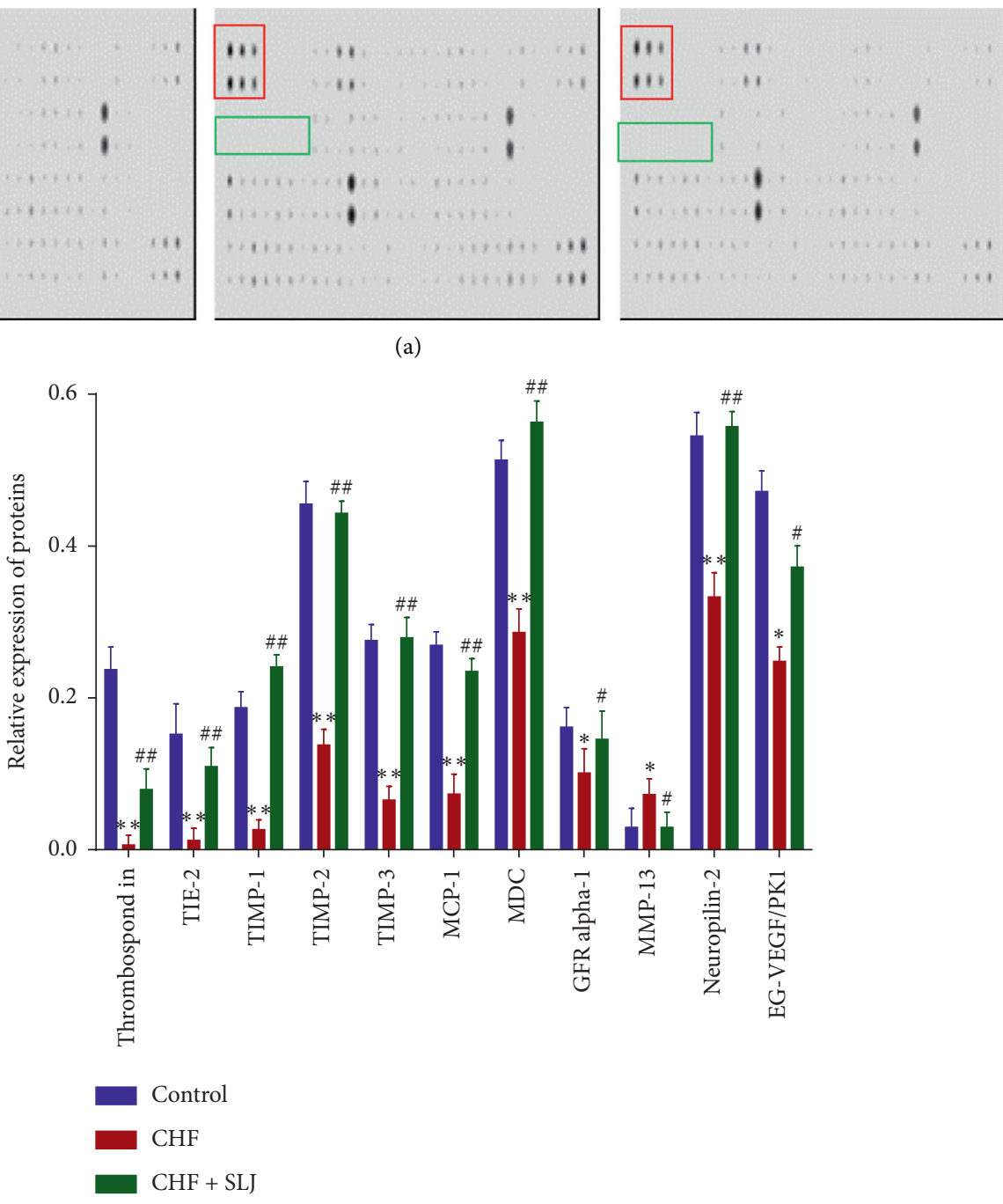

(b)

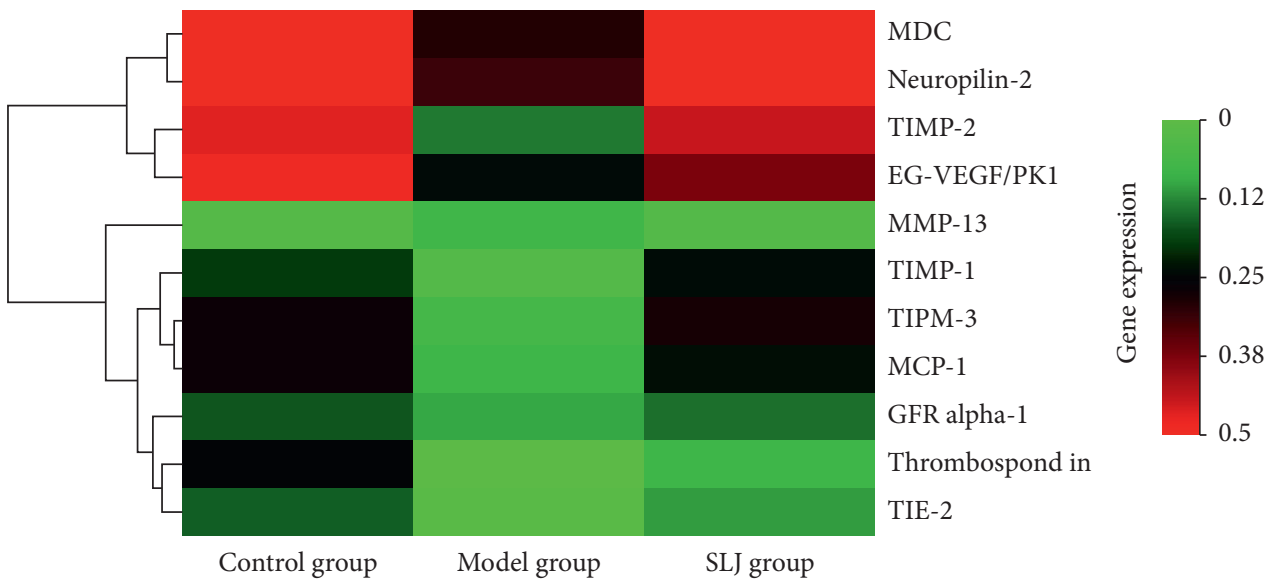

(c)

Figure 4: Analysis of antibody microarrays. (a) Image of protein chip data. Red box, positive control; green box, negative control. (b) A total of 11 proteins with significantly different expression levels were associated with SLJ treatment of CHF, as detected by protein array. (c) Heat map of protein microarray data reflecting the expression levels of the 11 proteins. Samples are indicated in columns and proteins in rows. Red, increased expression in SLJ treatment samples compared with CHF samples; green, decreased expression level; black, median expression. $n=3$. ${ }^{*} P<0.05$ and ${ }^{* *} P<0.01$ vs. the control group. ${ }^{\#} P<0.05$ and ${ }^{\# \#} P<0.01$ vs. the CHF group. SLJ, Shenlijia; CHF, chronic heart failure. 

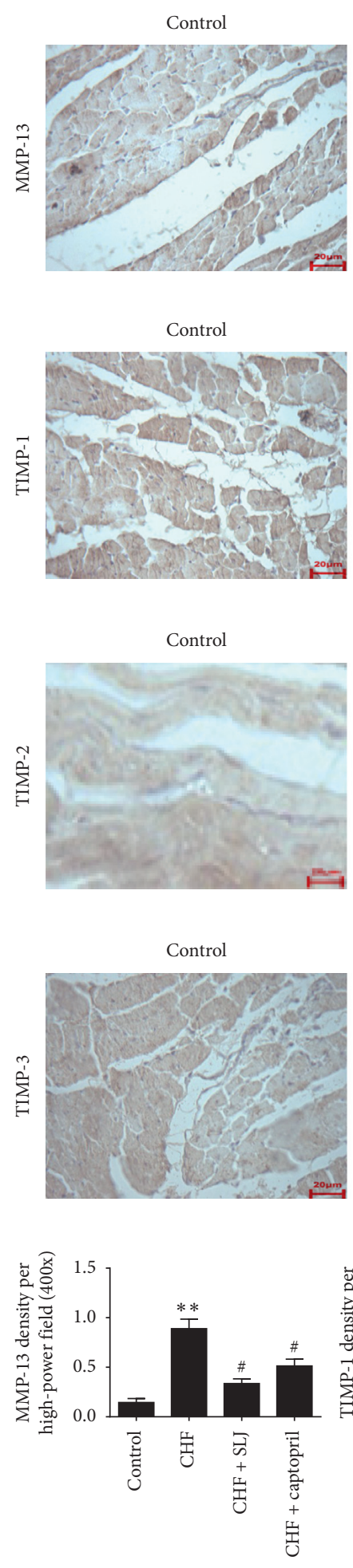

CHF

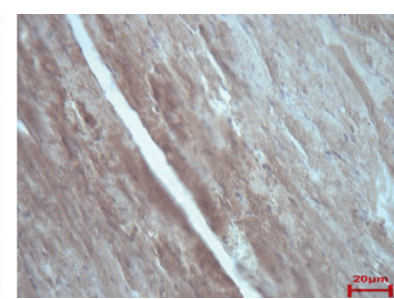

(a)
$\mathrm{CHF}+\mathrm{SLJ}$

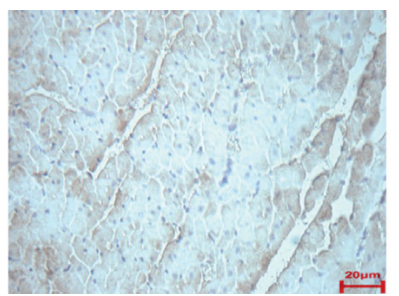

CHF + SLJ

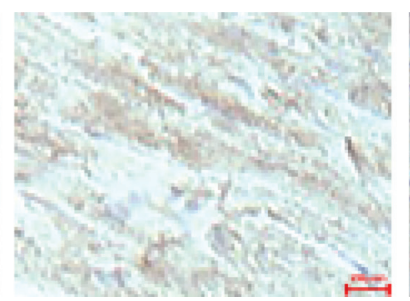

(b)

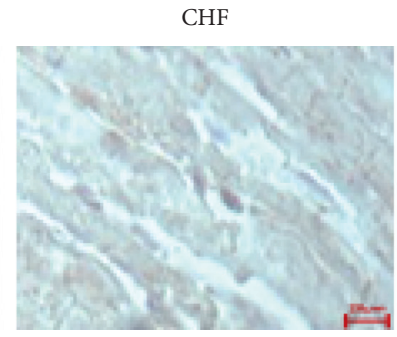

(c)
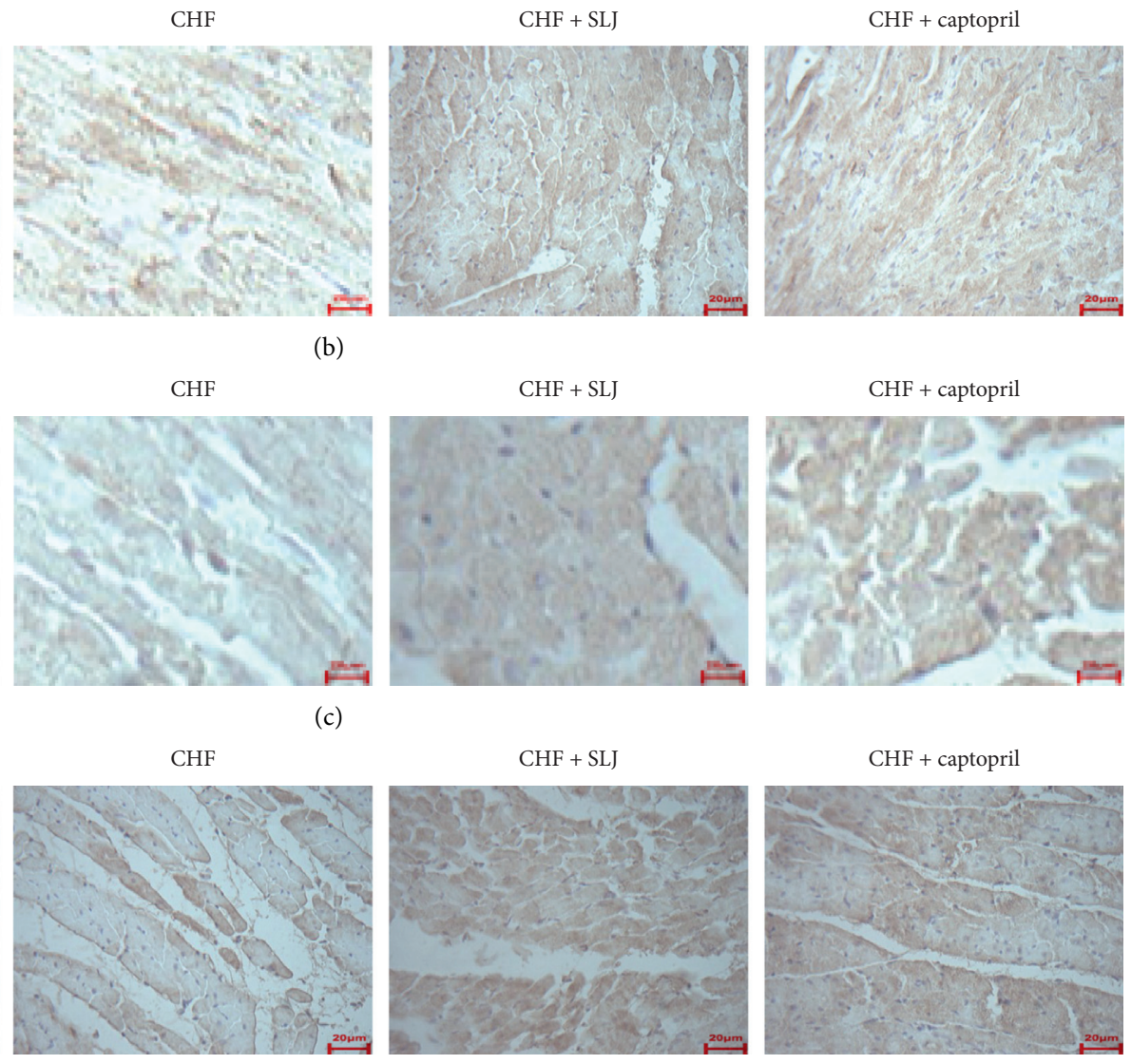

(d)
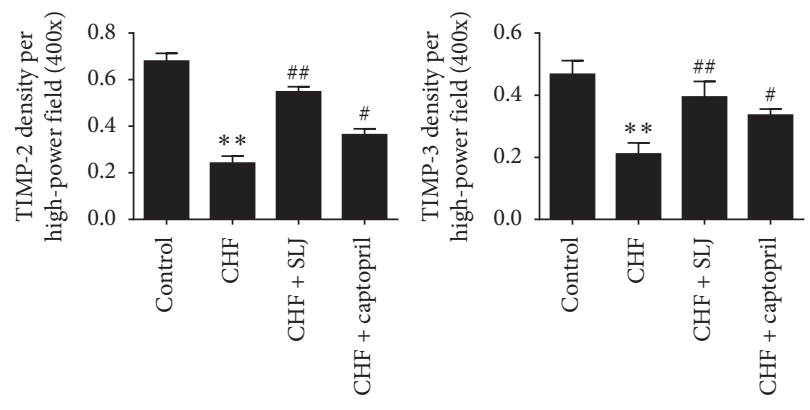

(e)

Figure 5: Continued. 


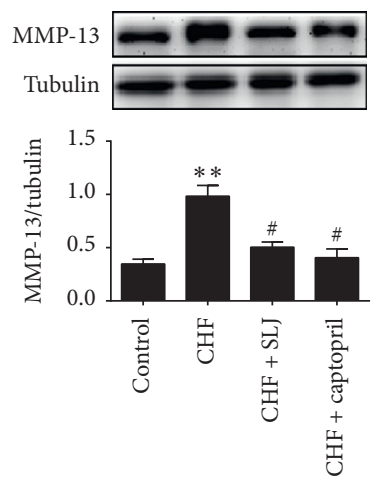

(f)

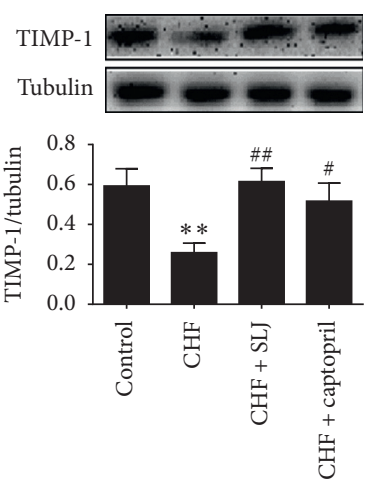

(g)

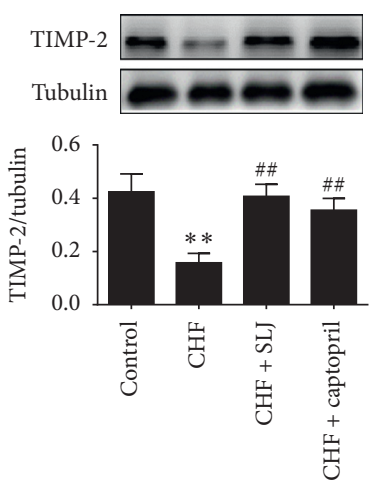

(h)

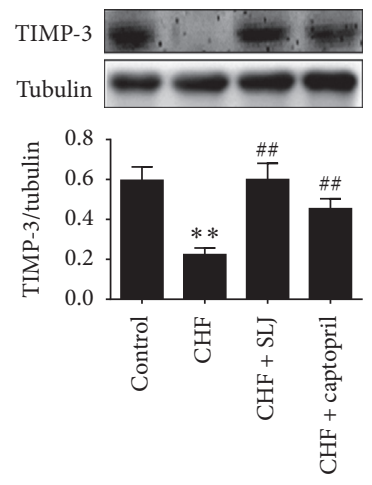

(i)

FIgURe 5: SLJ upregulates the expression of TIMP-1, TIMP-2, and TIMP-3 and downregulates that of MMP-13: (a-d) representative immunohistochemical image analyses of MMP-13, TIMP-1, TIMP-2, and TIMP-3 protein expressions; (e-h) western blot analysis of MMP13, TIMP-1, TIMP-2, and TIMP-3 protein expressions in myocardium. $n=8 .{ }^{*} P<0.05$ and ${ }^{* *} P<0.01$ vs. the control group. ${ }^{\#} P<0.05$ and ${ }^{\# \#} P<0.01$ vs. the CHF group. SJL, Shenlijia; TIMP, metalloproteinase inhibitor; MMP, matrix metalloproteinase; and CHF, chronic heart failure.

regulating the balance of TIMP-1, TIMP-2, TIMP-3, and MMP-13 expression.

\section{Discussion}

DOX is an effective anti-cancer drug with a wide therapeutic range. However, the clinical use of DOX is largely restricted by its side effects, which include cardiotoxicity, cardiac remodeling, and congestive heart failure [3]. Although DOX-induced cardiotoxicity and the pathophysiology of $\mathrm{CHF}$ have been widely researched, the underlying molecular mechanisms have not been confirmed, and there are few effective drugs available to prevent DOX-induced cardiopathologies clinically. In this regard, further research remains to be conducted. Nair S et al. found that cathepsin $\mathrm{K}$ may be a feasible drug target for the treatment of DOXinduced cardiotoxicity [29]. Based on the TCM theories on CHF therapy [30], the methodology has focused on using native compounds against DOX-induced cardiac toxicity. Previous studies have found that Chinese herbal medicines, date palm pollen extract and liensinine, can alleviate the DOX-induced cardiotoxicity [31, 32]. In the present study, the effects of SLJ, a new herbal compound, against DOXinduced CHF and its potential mechanisms were investigated in rats. The results showed that DOX administration caused heart failure and that SLJ exerted cardioprotection. The primary findings include the followings: (i) SLJ improved cardiac function; (ii) SLJ upregulated MCP-1, MDC, neuropilin-2, TGF- $\beta 3$, thrombospondin, TIE-2, EG-VEGF/ PK1, and TIMP-1/2/3 expression and downregulated that of MMP-13; and (iii) SLJ inhibited cardiac fibrosis through the TIMP-1, TIMP-2, and TIMP-3 or MMP-13 signaling pathway.

Some studies have shown that captopril (angiotensinconverting enzyme inhibitor) has a protective effect against DOX-induced cardiotoxicity and is commonly used as a reference drug in the study of Chinese herbal medicine for DOX-induced cardiotoxicity [33]. In the present study, the
CHF model was constructed using a $15 \mathrm{mg} / \mathrm{kg}$ DOX cumulative dose, and captopril was used as a reference drug to evaluate the protective effect of SLJ on DOX-induced heart failure. The hemodynamic parameters (that included $+\mathrm{dp} /$ dtmax, -dp/dtmax, and LVSP) were significantly lower, and LVEDP was markedly higher in the CHF group, compared with the control group; these effects were reversed by SLJ and captopril treatment. Moreover, echocardiographical results showed that SLJ and captopril treatment significantly increased EF and FS and markedly decreased LVIDd and LVIDs. These data provided a basis for the SLJ treatment of DOX-induced CHF.

Extracellular matrix (ECM) remodeling plays an important role in myocardial injury and repair, and regulatory ECM proteins are associated with the progression of various angiocardiopathies. Increased ECM deposition is a mark of cardiac hypertrophy, ischemic cardiomyopathy, and heart failure [34-37]. MMPs and TIMPs are the main regulators of the ECM. The causal relationship between the mediation and stimulation of MMPs and unfavorable alterations in cardiac structure and function has been clearly studied [38, 39]. Thus, regulating the induction and activation of MMPs and their inhibitors (TIMPs) may be an underlying target for the treatment of angiocardiopathy.

TIMPs consist of 4 unique molecules (TIMP-1-4) that differ in the time of expression after tissue damage and that may have a significant impact on the activity of MMPs and influence the proliferation and survival of fibroblasts. The first discovered TIMP, TIMP-1, was described in the late 1970s, and TIMP-4 was initially identified in the late 1990s; these proteins were initially considered to possess comparable structures that could bind to the active form of MMPs at a 1:1 stoichiometry ratio $[40,41]$. TIMPs may be involved in multiple cellular processes including propagation and apoptosis and inhibit MMP functioning [42]. MMPs comprise a diverse family of enzymes, including collagenases, stromelysins, gelatinases, and membrane prototype MMPs, all of which possess unique functionality. Multiple 
animal and clinical studies have uniformly demonstrated alterations and divergences in MMP and TIMP expressions and induction in myocardial remodeling $[43,44]$. In postmyocardial infarction remodeling, strong stimulation of MMPs was observed, specifically those linked with inflammation, though this was not necessarily accompanied by a corresponding increase in TIMPs. Specifically, the expressions of TIMP-1 and TIMP-2 increased at the early stages and were subsequently reduced following myocardial injury, while the relative TIMP-4 concentrations declined in the period directly after postmyocardial injury. By contrast, multiple MMPs are both robustly and persistently expressed [45]. Moreover, the increased inconsistency between MMP and TIMP levels is further instantiated when taking the stoichiometric ratio of MMP/TIMP into consideration, and the relative disorder between MMP and TIMP levels indicates the imbalance of this ECM proteolytic system following myocardial injury [46].

In the present study, TIMP-1, TIMP-2, and TIMP-3 expressions were found to be decreased, and MMP-13 expression was increased in the CHF group, compared with the control group, which was subsequently reversed by SLJ treatment. Moreover, compared with the control group, the expression levels of collagens I and III were increased in the CHF group, which was reduced by SLJ administration. These results suggest that SLJ protects the heart from DOX-associated toxicity by inhibiting myocardial fibrosis and remodeling at least partially via the TIMP-1/2/3 or MMP-13 signaling pathway.

To the best of our knowledge, the present study revealed for the first time that SLJ, a new TCM compound with multiple curative effects, significantly improves cardiac function and inhibits cardiac fibrosis, as evidenced by an upregulation in +dp/dtmax, -dp/dtmax, LVSP, EF, and FS and a downregulation in LVEDP, LVIDd, and LVIDs, regulating the balance of TIMP-1, TIMP-2, TIMP-3, and MMP-13. These results indicate that SLJ may be a potential new treatment for the prevention of DOX-induced cardiac damage. However, further research is required to confirm the potential molecular mechanisms involved.

\section{Data Availability}

The data sets used and/or analyzed during the current study are available from the corresponding author on reasonable request.

\section{Ethical Approval}

All experiments were performed according to the protocol approved by the Ethical Committee of Peking University First Hospital (Beijing).

\section{Conflicts of Interest}

The authors declare that they have no conflicts of interest.

\section{Authors' Contributions}

Deyou Jiang and Bowen Sui conceived and designed the study. Xutao Sun and Yunjia Song performed the experiments and analyzed the data. Ying Xie, Jieru Han, and Yang Sun contributed to the acquisition and interpretation of data. Fei Chen discussed the results. Xutao Sun drafted the manuscript with support from Fei Chen. All authors critically revised the work and approved the final manuscript. Xutao Sun and Yunjia Song contributed equally to this study.

\section{Acknowledgments}

This study was supported by the Heilongjiang University of Chinese Medicine Foundation (2019BS06) and the Postdoctoral Foundation of Heilongiang Province (LBHZ19035).

\section{References}

[1] J. R. Mackey, M. Martin, T. Pienkowski et al., "Adjuvant docetaxel, doxorubicin, and cyclophosphamide in nodepositive breast cancer: 10-year follow-up of the phase 3 randomised BCIRG 001 trial," The Lancet Oncology, vol. 14, pp. 72-80, 2013.

[2] M. Mazevet, M. Moulin, A Llach-Martinez et al., "Complications of chemotherapy, a basic science update," La Presse médicale, vol. 42, pp. e352-e361, 2013.

[3] S. E. Lipshultz, T. L. Miller, R. E. Scully et al., "Changes in cardiac biomarkers during doxorubicin treatment of pediatric patients with highrisk acute lymphoblastic leukemia: associations with long-term echocardiographic outcomes," Journal of Clinical Oncology, vol. 30, pp. 1042-1049, 2012.

[4] A. Briasoulis, E. Androulakis, T. Christophides, and D. Tousoulis, "The role of inflammation and cell death in the pathogenesis, progression and treatment of heart failure," Heart Failure Reviews, vol. 21, pp. 169-176, 2016.

[5] R. Moudgil and E. T. Yeh, "Mechanisms of cardiotoxicity of cancer chemotherapeutic agents: cardiomyopathy and beyond," The Canadian Journal of Cardiology, vol. 32, pp. 863-870, 2016.

[6] M. Nian, P. Lee, N. Khaper, and P. Liu, "Inflammatory cytokines and post myocardial infarction remodeling," Circulation Research, vol. 94, pp. 1543-1553, 2004.

[7] G. C. Meléndez, J. H. Jordan, R. B. D’Agostino, S. Vasu, C. A. Hamilton, and W. G. Hundley, "Progressive 3-month increase in LV myocardial ECV after anthracycline-based chemotherapy," JACC Cardiovasc Imaging, vol. 10, pp. 708-709, 2016.

[8] J. H. Jordan, S. Vasu, T. M. Morgan et al., "Anthracyclineassociated $\mathrm{t} 1$ mapping characteristics are elevated independent of the presence of cardiovascular comorbidities in cancer survivors," Circulation: Cardiovascular Imaging, vol. 3, no. 2, Article ID e004325, 2016.

[9] K. L. Nguyen, P. Hu, D. B. Ennis, J. Shao, K. A. Pham, and J. J. Chen, "Cardiac MRI: a translational imaging tool for characterizing anthracycline-induced myocardial remodeling," Current Oncology Reports, vol. 18, p. 48, 2016.

[10] W. H. W. Tang and Y. Huang, "Cardiotonic modulation in heart failure: insights from traditional Chinese medicine," Journal of the American College of Cardiology, vol. 62, pp. 1073-1074, 2013.

[11] P. Hao, F. Jiang, J. Cheng, L. Ma, Y. Zhang, and Y. Zhao, "Traditional Chinese medicine for cardiovascular disease: evidence and potential mechanisms," Journal of the American College of Cardiology, vol. 69, pp. 2952-2966, 2017. 
[12] D. Liang and M. Zhang, "The thinking on TCM differential treatment of congestive heart failure," Journal of Traditional Chinese Medical Sciences, vol. 20, pp. 44-47, 2000.

[13] 2010 Chinese pharmacopoeia, part I.

[14] L. Li, J. Ni, M. Li et al., "Ginsenoside Rg3 micelles mitigate doxorubicin-induced cardiotoxicity and enhance its anticancer efficacy," Drug Delivery, vol. 24, pp. 1617-1630, 2017.

[15] X. Yan, H. Wu, J Ren et al., "Shenfu Formula reduces cardiomyocyte apoptosis in heart failure rats by regulating microRNAs," Journal of Ethnopharmacology, vol. 227, pp. 105-112, 2018.

[16] S. Zhang, L. Zhang, H Zhang et al., "Hongjingtian injection attenuates myocardial oxidative damage via promoting autophagy and inhibiting apoptosis," Oxidative Medicine and Cellular Longevity, vol. 2017, Article ID 6965739, 11 pages, 2017.

[17] L. C. Wang, W. S. Zhang, Q. Liu, J. Li, R. N. Alolga, and K. Liu, "A standardized notoginseng extract exerts cardioprotection by attenuating apoptosis under endoplasmic reticulum stress conditions," The Journal of Functional Foods, vol. 16, pp. 20-27, 2015.

[18] Z. R. Ge, M. C. Xu, Y. Huang, C. J. Zhang, J. Lin, and C. W. Ruan, "Cardioprotective effect of notoginsenoside R1 in a rabbit lung remote ischemic postconditioning model via activation of the TGF-beta 1/TAK1 signaling pathway," Experimental and Therapeutic Medicine, vol. 11, pp. 2341-2348, 2016.

[19] S. Fu, J. Zhang, X Gao et al., "Clinical practice of traditional Chinese medicines for chronic heart failure," Heart Asia, vol. 2, pp. 24-27, 2010.

[20] T. Miyawaki, H. Aono, Y. Toyoda-Ono, H. Maeda, Y. Kiso, and K. Moriyama, "Antihypertensive effects of sesamin in humans," Journal of Nutritional Science and Vitaminology, vol. 55, pp. 87-91, 2009.

[21] J. Lee, D. S. Jang, N. H. Kim, Y. M. Lee, J. Kim, and J. S. Kim, "Galloyl glucoses from the seeds of cornus officinalis with inhibitory activity against protein glycation, aldose reductase, and cataractogenesis ex vivo," Biological and Pharmaceutical Bulletin, vol. 34, pp. 443-446, 2011.

[22] B. H. Chung, J. D. Kim, C. K Kim et al., "Icariin stimulates angiogenesis by activating the MEK/ERK- and PI3K/Akt/ eNOS-dependent signal pathways in human endothelial cells," Biochemical and Biophysical Research Communications, vol. 376, pp. 404-408, 2008.

[23] H. F. Zhang, L. H. Yan, Q. W. Zhang, and Z. M. Wang, "Flavonoids from leaves of Epimedium pubescens," Chinese Medical Journal, vol. 38, pp. 1942-1946, 2013.

[24] Y. H. Song, H. Cai, N. Gu, C. F. Qian, S. P. Cao, and Z. M. Zhao, "Icariin attenuates cardiac remodelling through down-regulating myocardial apoptosis and matrix metalloproteinase activity in rats with congestive heart failure," Journal of Pharmacy and Pharmacology, vol. 63, pp. 541-549, 2011.

[25] M. L. Xu, Z. Y. Zheng, Y. J Xia et al., "Shexiang baoxin pill, a formulated Chinese herbal mixture, induces neuronal differentiation of PC12 cells: a signaling triggered by activation of protein kinase A," Frontiers in Pharmacology, vol. 10, p. 1130, 2019.

[26] X. Sun, G. Chen, Y Xie et al., "Qiliqiangxin improves cardiac function and attenuates cardiac remodelling in doxorubicininduced heart failure rats," Pharmaceutical Biology, vol. 58, no. 1, pp. 417-426, 2020.
[27] N. Tanaka, N. Dalton, L Mao et al., "Transthoracic echocardiography in models of cardiac disease in the mouse," Circulation, vol. 94, no. 5, pp. 1109-1117, 1996.

[28] Y. Song, H. Peng, D. Bu et al., "Negative auto-regulation of sulfur dioxide generation in vascular endothelial cells: AAT1 S-sulfenylation," Biochemical and Biophysical Research Communications, vol. 19, pp. S0006-291X, 2020.

[29] X. Liang, S. Wang, L. Wang, A. F. Ceylan, J. Ren, and Y. Zhang, "Mitophagy inhibitor liensinine suppresses doxorubicin-induced cardiotoxicity through inhibition of Drp1-mediated maladaptive mitochondrial fission," Pharmacological Research, vol. 157, Article ID 104846, 2020.

[30] M. A. Vasilopoulou, E. Ioannou, V. Roussis, and N. Chondrogianni, "Modulation of the ubiquitin-proteasome system by marine natural products," Redox Biology, vol. 41, Article ID 101897, 2021.

[31] R. Guo, Y. Hua, J. Ren, K. E. Bornfeldt, and S. Nair, "Cardiomyocyte-specific disruption of Cathepsin $\mathrm{K}$ protects against doxorubicin-induced cardiotoxicity," Cell Death \& Disease, vol. 10, no. 12, p. 933, 2019.

[32] S. S. Elblehi, Y. S. El-Sayed, M. M. Soliman, and M. Shukry, "Date palm pollen extract avert doxorubicin-induced cardiomyopathy fibrosis and associated oxidative/nitrosative stress, inflammatory cascade, and apoptosis-targeting bax/ bcl-2 and caspase-3 signaling pathways," Animals (Basel), vol. 11, no. 3, p. 886, 2021.

[33] R. Wu, P. A. Yao, H. L Wang et al., "Effect of fermented Cordyceps sinensis on doxorubicin-induced cardiotoxicity in rats," Molecular Medicine Reports, vol. 18, no. 3, pp. 3229-3241, 2018.

[34] B. C. Berk, K. Fujiwara, and S. Lehoux, "ECM remodeling in hypertensive heart disease," Journal of Clinical Investigation, vol. 117, pp. 568-575, 2007.

[35] B. L. Leonard, B. H. Smaill, and I. J. LeGrice, "Structural remodeling and mechanical function in heart failure," $\mathrm{Mi}$ croscopy and Microanalysis, vol. 18, pp. 50-67, 2012.

[36] D. Fan, A. Takawale, J. Lee, and Z. Kassiri, "Cardiac fibroblasts, fibrosis and extracellular matrix remodeling in heart disease," Fibrogenes Tissue Repair, vol. 5, no. 15, 2012.

[37] A. F. Bayomy, M. Bauer, Y. Qiu, and R. Liao, "Regeneration in heart disease-Is ECM the key?" Life Science, vol. 91, pp. 823-827, 2012.

[38] J. T. Peterson, H. Hallak, L. Johnson et al., "Matrix metalloproteinase inhibition attenuates left ventricular remodeling and dysfunction in a rat model of progressive heart failure," Circulation, vol. 103, pp. 2303-2309, 2011.

[39] G. Dormán, S. Cseh, I. Hajdú et al., "Matrix metalloproteinase inhibitors: a critical appraisal of design principles and proposed therapeutic utility," Drugs, vol. 70, pp. 949-964, 2010.

[40] E. A. Bauer, G. P. Stricklin, J. J. Jeffrey, and A. Z. Eisen, "Collagenase production by human skin fibroblasts," Biochemical and Biophysical Research Communications, vol. 64, pp. 232-240, 1975.

[41] J. Greene, M. Wang, Y. E. Liu, L. A. Raymond, C. Rosen, and Y. E. Shi, "Molecular cloning and characterization of human tissue inhibitor of metalloproteinase 4," Journal of Biological Chemistry, vol. 271, pp. 30375-30380, 1996.

[42] R. Visse and H. Nagase, "Matrix metalloproteinases and tissue inhibitors of metalloproteinases: structure, function, and biochemistry," Circulation Research, vol. 92, pp. 827-839, 2003.

[43] R. P. Iyer, N. L. Patterson, G. B. Fields, and M. L. Lindsey, “The history of matrix metalloproteinases: milestones, myths, and 
misperceptions," The American Journal of Physiology-Heart and Circulatory Physiology, vol. 303, pp. H919-H930, 2012.

[44] K. Konstantinidis, R. S. Whelan, and R. N. Kitsis, "Mechanisms of cell death in heart disease," Arteriosclerosis, Thrombosis, and Vascular Biology, vol. 32, pp. 1552-1562, 2012.

[45] E. M. Wilson, S. L. Moainie, J. M. Baskin et al., "Region- and type-specific induction of matrix metalloproteinases in postmyocardial infarction remodeling," Circulation, vol. 107, pp. 2857-2863, 2003.

[46] C. S. Webb, D. D. Bonnema, S. H. Ahmed et al., "Specific temporal profile of matrix metalloproteinase release occurs in patients after myocardial infarction: relation to left ventricular remodeling," Circulation, vol. 114, pp. 1020-1027, 2006. 